SMALL INTESTINE

\title{
Oats to children with newly diagnosed coeliac disease: a randomised double blind study
}

\author{
L Högberg, P Laurin, K Fälth-Magnusson, C Grant, E Grodzinsky, G Jansson, H Ascher, \\ L Browaldh, J-Å Hammersjö, E Lindberg, U Myrdal, L Stenhammar
}

Gut 2004;53:649-654. doi: 10.1136/gut.2003.026948

See end of article for authors' affiliations

Correspondence to: Dr L Högberg, Department of Paediatrics, Linköping University, Norrköping Hospital, SE-601 82 Norrköping, Sweden; lotta.hogberg@lio.se

Accepted for publication 12 November 2003
Background: Treatment of coeliac disease (CD) requires lifelong adherence to a strict gluten free diet (GFD) which hitherto has consisted of a diet free of wheat, rye, barley, and oats. Recent studies, mainly in adults, have shown that oats are non-toxic to CD patients. In children, only open studies comprising a small number of patients have been performed.

Aim: To determine if children with CD tolerate oats in their GFD.

Patients and methods: In this double blind multicentre study involving eight paediatric clinics, 116 children with newly diagnosed CD were randomised to one of two groups: one group was given a standard GFD (GFD-std) and one group was given a GFD with additional wheat free oat products (GFD-oats). The study period was one year. Small bowel biopsy was performed at the beginning and end of the study. Serum IgA antigliadin, antiendomysium, and antitissue transglutaminase antibodies were monitored at $0,3,6$, and 12 months.

Results: Ninety three patients completed the study. Median (range) daily oat intake in the GFD-oats group $(\mathrm{n}=42)$ was $15(5-40) \mathrm{g}$ at the six month control and $15(0-43) \mathrm{g}$ at the end of the study. All patients were in clinical remission after the study period. The GFD-oats and GFD-std groups did not differ significantly at the end of the study regarding coeliac serology markers or small bowel mucosal architecture, including numbers of intraepithelial lymphocytes. Significantly more children in the youngest age group withdrew. Conclusions: This is the first randomised double blind study showing that the addition of moderate amounts of oats to a GFD does not prevent clinical or small bowel mucosal healing, or humoral immunological downregulation in coeliac children. This is in accordance with the findings of studies in adult coeliacs and indicates that oats, added to the otherwise GFD, can be accepted and tolerated by the majority of children with $C D$.
1 $\mathrm{n}$ coeliac disease $(\mathrm{CD})$ there is an enteropathy affecting mainly the proximal small bowel mucosa of genetically predisposed individuals. In 1950, Dicke ${ }^{1}$ was the first to report that symptoms and signs of $\mathrm{CD}$ in children disappeared when wheat and rye were removed from the diet. Wheat, rye, and barley are closely related cereals of the family Triticeae and are toxic to patients with $\mathrm{CD}^{2}$ Oats can be classified as a cousin of Triticeae and thus more distantly related. Prolamins, which comprise the alcohol soluble fraction of the storage proteins of cereals, are termed gliadin in wheat and avenin in oats. They have been named according to their content of the amino acids proline and glutamine, which is much higher in gliadin than in avenin. ${ }^{2}$ The proportion of prolamins in cereals may reflect their toxicity to coeliacs. However, recent studies of supposedly toxic peptides in prolamins have not revealed any of these amino acid sequences in oats. ${ }^{34}$

Early studies on the toxicity of oats in patients with CD have yielded contrasting results, ${ }^{56}$ while most recent studies ${ }^{7-9}$ report that oats are non-toxic. Previous studies in children however have been open, comprising a comparatively small number of patients. To shed further light on whether or not oats are non-toxic to children with $\mathrm{CD}$, we performed the present study, which is the first randomised double blind study on the toxicity of oats in a large population of coeliac children.

\section{METHODS}

\section{Study design}

This randomised, double blind, multicentre study which started in April 1998 and involved eight Swedish paediatric clinics in Norrköping, Linköping, Motala, Västervik, Västerås, Örebro, Stockholm (Sachsska Hospital), and Göteborg. The last patient was included in September 2001.

\section{Patients}

A total of 116 children with newly diagnosed symptomatic CD were included. Criteria for inclusion, apart from willingness to participate, were: age less than 18 years; small bowel biopsy showing enteropathy; and a good understanding of the Swedish language. Mean age was 6.5 years (SD 4.6; median 6.0; range 8 months-17.5 years). The male:female distribution was equal in the two groups (1:1.4).

The 116 children included were randomised to a standard gluten free diet (GFD) with (GFD-oats; $n=57$ ) or without (GFD-std; $n=59$ ) oats (fig 1 ). If there was more than one participant in a family, the sibling was included in the same group as the first participant. Each child was studied over a period of one year. The study diet was started within two weeks from the first diagnostic biopsy.

\section{Associated diseases}

Seven children had diabetes mellitus, of whom three were in the GFD-oats group; six other patients had IgA deficiency,

Abbreviations: $A G A$, antigliadin antibodies; $C D$, coeliac disease; EMA, antiendomysium antibodies; GFD, gluten free diet; GFD-oats, gluten free diet including oats; GFD-std, standard gluten free diet; TGA, antitissue transglutaminase antibodies; PBS, phosphate buffered saline; IEL, intraepithelial lymphocytes 


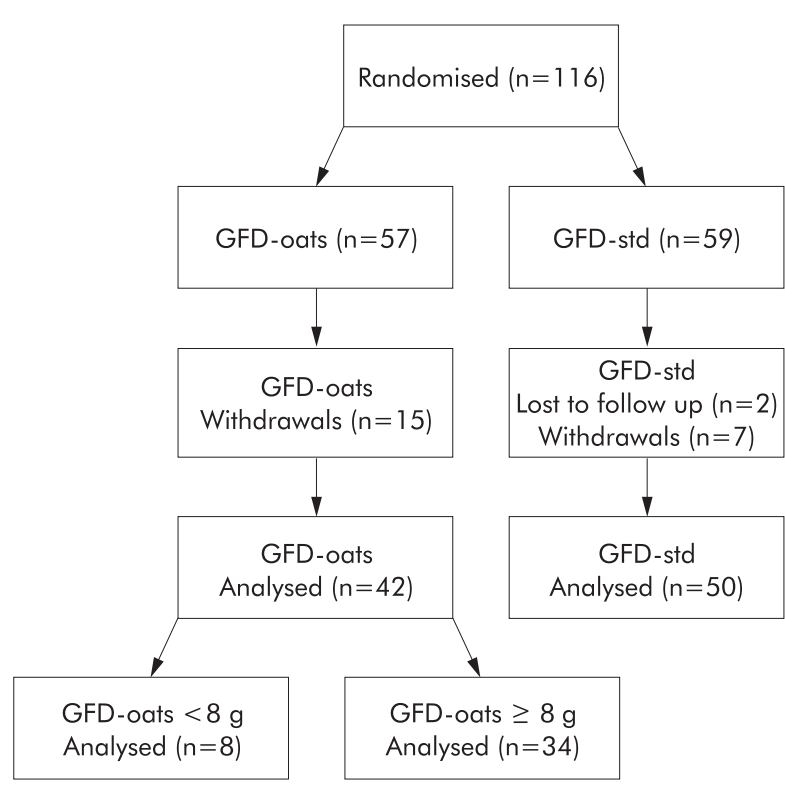

Figure 1 Flow chart of the study groups. GFD-oats, gluten free diet including oats; GFD-std, standard gluten free diet; GFD-oats $<8 \mathrm{~g}$, gluten free diet including less than $8 \mathrm{~g}$ of oats daily at the end of the study; GFD-oats $\geqslant 8 \mathrm{~g}$, gluten free diet including at least $8 \mathrm{~g}$ of oats daily at the end of the study.

only one of them in the GFD-oats group. The only patient with Down's syndrome had GFD without oats.

\section{Study diet}

The oats used were specially grown, milled, and packaged so as not to become contaminated with wheat, rye, or barley (manufacturer Semper AB, Sweden: now commercially available on the Swedish market). Furthermore, the oat products were tested by an ELISA assay (Transia Gluten ELISA; Diffchamb, Sweden) to ensure absence of gluten contamination. To ensure blinding of the diet, oats were mixed with otherwise gluten free products, such as mixes for formula, porridge and bread, baked ready made bread, and cookies. We aimed at a daily oat intake of 25-50 g. The parents of each study patient were requested to monitor the daily intake of study products for the first month of the diet and thereafter for one week immediately prior to visits to the clinic at 3, 6, 9, and 12 months, respectively.

\section{Examinations}

Blood samples were taken at 0, 3, 6, and 12 months. Sera were stored at $-20^{\circ} \mathrm{C}$ pending analysis. IgA antigliadin antibody (AGA), antiendomysium antibody (EMA), and antitissue transglutaminase (TGA) titres were measured. As selective $\operatorname{Ig} A$ deficiency is more common in $\mathrm{CD}^{10}{ }^{11}$ and may give a false negative result on screening, total IgA titre was also measured. When a low IgA value was found, measurement of IgG EMA titre was also done. After approximately one (mean 1.1 years; range 0.9-1.5) year on the GFD with or without oats, a small bowel biopsy was done to assess healing of the mucosa.

\section{Antigliadin antibodies}

An ELISA was used for detection of IgA AGA. ${ }^{12}$ Microtitre plates were coated with $50 \mu \mathrm{g} / \mathrm{ml}$ gliadin dissolved in ethanol (Sigma, St Louis, Missouri, USA). Plates were incubated for 60 minutes with negative and low/high positive control titrated reference serum, and patient sera were diluted 1 in 10 in phosphate buffered saline (PBS) containing 0.5\% human serum albumin and then washed. Peroxidase conjugated antihuman IgA antibodies (Dakopatts, Glostrup, Denmark) were used to detect IgA AGA. Absorbance was read spectrophotometrically in a Dynatech minireader II (Dynatech, Alexandria, Virginia, USA) and set against the titrated positive serum. Optical density of the reference was transformed to units using a program (Dynatech) for semilog fit. The cut off for a positive outcome was 30 units. ${ }^{13}$

\section{Antiendomysium antibodies}

IgA and IgG EMA titres were measured by indirect immunofluorescence microscopy using fixed cryostat sections of monkey oesophagus (Biosystems, Barcelona, Spain) as an antigen substrate. ${ }^{14}{ }^{15}$ Patient sera were screened at a dilution of 1 in 10 in PBS, pH 7.4, together with a positive and negative control. Polyclonal fluorescein isothiocyanate labelled rabbit antihuman IgA or IgG (Dakopatts) was used as the secondary antibody. Positive sera $\geqslant 1 / 10$ were then end point titrated. The antibody titre was defined as the highest serum dilution yielding positive fluorescence.

\section{Antitissue transglutaminase antibodies}

IgA TGA titre was measured using the commercially available ELISA kit Celikey (Pharmacia Diagnostics AB, Freiburg, Germany) with human recombinant tissue transglutaminase as the antigen. Microtitre wells coated with recombinant human tTG were incubated with $100 \mu \mathrm{l}$ of serum diluted $1: 101$. Bound IgA was detected with horseradish peroxidase labelled rabbit antihuman IgA.3,3',5,5' Tetramethylbenzidine was used for colour development and absorbance was measured at $450 \mathrm{~nm}$ with a reference wavelength of $620 \mathrm{~nm}$. Total incubation time for the ELISA was 70 minutes. A positive and negative control and a six point calibrator curve were included in each assay run. The calibrator curve, containing IgA TGA from 0 to $100 \mathrm{U} / \mathrm{ml}$, was used for determination of antibody levels in the patient samples. The manufacturer classed the results as negative when $<5 \mathrm{U} / \mathrm{ml}$, equivocal $5-8 \mathrm{U} / \mathrm{ml}$, and positive $>8 \mathrm{U} / \mathrm{ml}$.

\section{Total serum IgA}

All sera were analysed for total serum $\operatorname{IgA}$ using a routine nephelometric assay. If levels were below detection $(<0.05 \mathrm{~g} / \mathrm{l})$, IgG EMA titre was measured as described above.

\section{Small bowel biopsy}

At five clinics, a small bowel biopsy was performed by peroral suction capsule obtaining 1-2 specimens from the distal duodenum or proximal jejunum. At the remaining three clinics, the majority of biopsies were obtained endoscopically with at least three mucosal samples from the distal duodenum.

All mucosal biopsy specimens were examined by a local pathologist at each hospital. Specimens were re-examined by an experienced paediatric pathologist, blinded both to sequence, initial or repeat, and to dietary regimen. All biopsies were routinely fixed in $10 \%$ formalin for preparation of conventional paraffin sections with haematoxylin and eosin as the standard stain.

For the purposes of this review, duodenal biopsies were assessed as being either compatible with gluten enteropathy-partial, subtotal, or total villous atrophy with concomitant changes among enterocytes and in the lamina propria, as in the Marsh classification type $3^{16}$-or as architecturally intact (that is, restored morphologically). Counts of intraepithelial lymphocytes (IEL) were done on all biopsy specimens with a restored mucosa structure. Only stretches where enterocytes were sectioned perpendicularly or nearly so to the basal membrane were counted. In practice, this gave an upper limit of 300 enterocytes per biopsy. Three sets of 100 


\begin{tabular}{|c|c|c|c|}
\hline & GFD-oats $(n=15)$ & GFD-std $(n=7)$ & p Value \\
\hline Age at first biopsy (y) (median (range)) & $3.3(0.8-17.5)$ & $8.3(1.1-10.7)$ & 0.94 \\
\hline Patients $<2$ y (n (\%)) & $4(27 \%)$ & $2(29 \%)$ & $>0.99$ \\
\hline Sex distribution (M:F) & $1: 1.5$ & $1: 2.5$ & $>0.99$ \\
\hline No symptoms & 9 & 5 & \\
\hline Symptoms & 6 & 2 & $>0.99$ \\
\hline
\end{tabular}

enterocytes each from each biopsy were counted and the mean IEL count was taken to the nearest whole number.

\section{Statistical analysis}

All analyses were carried out according to the per protocol principle. Statistical analyses were performed using StatView software (StatView; SAS Institute Inc., Cary, North Carolina, USA). Non-parametric tests were used when variables were not normally distributed and did not have equal variance. Results are presented as mean (SD) or median (range). Differences between groups were assessed by the Student's test (two tailed $t$ test) or the Mann-Whitney $\mathrm{U}$ test. The $\chi^{2}$ test (two tailed) was used to analyse differences in the frequency between the study groups.

\section{Ethics}

The study was approved by the Human Research Ethics Committee of the Faculty of Health Sciences, University of Linköping, as the main ethics committee for the investigation.

\section{RESULTS}

\section{Withdrawals}

Twenty two of 116 children withdrew from the study (tables 1, 2; fig 1). The reasons for this varied. The most common reason was inability to follow the diet. Eight children did not do well on the diet and continued on a conventional gluten free diet. Among patients in the GFDoats group, 15 of $57(26 \%)$ withdrew, six because of symptoms. One eight year old boy in the GFD-oats group had no catch up in growth during the first four months of a GFD. He was withdrawn from the study by his local paediatrician and put on a conventional GFD.. The symptoms described in the remaining five patients were abdominal pain and/or diarrhoea. In the GFD-std group, seven of 59 (11\%) patients withdrew from the study. Two had gastrointestinal symptoms. The difference in withdrawal frequency in the two groups was not significant $(\mathrm{p}=0.08)$. Two patients could not be evaluated because they did not have a control biopsy performed after one year on the diet. Both were in the GFDstd group. The remaining 92 patients completed the study (table 3).

\section{Amount of oats ingested}

Median (range) amount of oats ingested in the GFD-oats group $(\mathrm{n}=42)$ was $15(5-40) \mathrm{g}$ at the six month control and $15(0-43) \mathrm{g}$ at the 12 month control (= time of control biopsy).

In the GFD-oats group, some children consumed very small amounts of oat products towards the end of the study year. For that reason we divided the GFD-oats patients into two subgroups according to the amount of oats ingested at the end of the study year: children taking at least $8 \mathrm{~g}$ of oats daily $(\mathrm{n}=34)$ and children taking less than $8 \mathrm{~g}$ daily $(\mathrm{n}=8)$. (For explanation of the choice of the smallest amount of $8 \mathrm{~g}$ of oats daily, see under discussion section.)

The subgroup of study patients consuming at least $8 \mathrm{~g}$ of oats daily (GFD-oats $\geqslant 8 \mathrm{~g}$ ) at the end of the study was also compared with the GFD-std group (table 3 ). In this subgroup, median intake of oats was $17.5(5-40) \mathrm{g}$ and $20(8-43) \mathrm{g}$ at six and 12 months, respectively. Differences in outcome between the GFD-oats and GFD-std groups or between the GFD-oats $\geqslant 8 \mathrm{~g}$ and GFD-std groups were not significant (table 3).

\begin{tabular}{|c|c|c|c|c|}
\hline $\begin{array}{l}\text { Age at study } \\
\text { start (y) }\end{array}$ & $\begin{array}{l}\text { Sex } \\
(M / F)\end{array}$ & $\begin{array}{l}\text { Study diet (GFD-oats/ } \\
\text { GFD-std) }\end{array}$ & $\begin{array}{l}\text { Duration of study } \\
\text { diet (weeks) }\end{array}$ & $\begin{array}{l}\text { Withdrawal due to suspected } \\
\text { intolerance to study diet (yes } / \mathrm{no} \text { ) }\end{array}$ \\
\hline 0.8 & M & GFD-oats & 1 & Yes \\
\hline 1.2 & M & GFD-oats & 4 & Yes \\
\hline 1.2 & M & GFD-oats & 12 & No \\
\hline 1.2 & $\mathrm{~F}$ & GFD-oats & 12 & Yes \\
\hline 2.1 & $\mathrm{~F}$ & GFD-oats & 4 & No \\
\hline 2.4 & $\mathrm{~F}$ & GFD-oats & 6 & Yes \\
\hline 2.4 & $\mathrm{~F}$ & GFD-oats & 3 & No \\
\hline 3.3 & $\mathrm{~F}$ & GFD-oats & 8 & Yes \\
\hline 6.2 & $\mathrm{~F}$ & GFD-oats & 26 & No \\
\hline 7.3 & $\mathrm{~F}$ & GFD-oats & 8 & No \\
\hline 8.0 & M & GFD-oats & 20 & Yes \\
\hline 10.7 & M & GFD-oats & 3 & No \\
\hline 15.1 & M & GFD-oats & 4 & No \\
\hline 17.3 & $\mathrm{~F}$ & GFD-oats & 12 & No \\
\hline 17.5 & $\mathrm{~F}$ & GFD-oats & 24 & No \\
\hline 1.1 & $\mathrm{~F}$ & GFD-std & 2 & Yes \\
\hline 1.2 & M & GFD-std & 6 & Yes \\
\hline 2.7 & $\mathrm{~F}$ & GFD-std & 22 & No \\
\hline 8.3 & $\mathrm{~F}$ & GFD-std & 12 & No \\
\hline 9.6 & M & GFD-std & 40 & No \\
\hline 9.3 & $\mathrm{~F}$ & GFD-std & 1 & No \\
\hline 10.7 & $\mathrm{~F}$ & GFD-std & 10 & No \\
\hline
\end{tabular}


Table 3 Details of patients completing the study and results of some examinations

\begin{tabular}{|c|c|c|c|c|}
\hline & $\begin{array}{l}\text { GFD-oats } \\
(n=42)\end{array}$ & $\begin{array}{l}\text { GFD-oats } \geqslant 8 \mathrm{~g} \\
(\mathrm{n}=34)\end{array}$ & $\begin{array}{l}\text { GFD-std } \\
(n=50)\end{array}$ & p Value* \\
\hline Age at inclusion (y) (median (range)) & $7.2(0.7-15.7)$ & $7.2(0.7-15.7)$ & $5.6(0.8-17.2)$ & $0.37 ; 0.38$ \\
\hline Patients $<2$ y $(\mathrm{n}(\%))$ & $11(26 \%)$ & $9(26 \%)$ & $12(24 \%)$ & $>0.99 ;>0.99$ \\
\hline Sex distribution (M:F) & $1: 1.3$ & $1: 1.0$ & $1: 1.3$ & $>0.99 ; 0.75$ \\
\hline Length of diet (y) (median (range)) & $1.1(0.9-1.3)$ & $1.1(0.9-1.2)$ & $1.1(0.9-1.5)$ & $0.47 ; 0.80$ \\
\hline Enteropathy at end of study period (n) & 0 & 0 & 2 & $0.50 ; 0.51$ \\
\hline IEL count (per 100 enterocytes) (mean (SD) [range]) & $16(4.5)[6-26]$ & $16(4.0)[9-24]$ & $16(5.0)[3-29]$ & $0.84 ; 0.94$ \\
\hline EMA positive at control biopsy (\%) & 33 & 35 & 24 & $0.45 ; 0.38$ \\
\hline TGA positive at control biopsy (\%) & 17 & 21 & 10 & $0.49 ; 0.27$ \\
\hline
\end{tabular}

GFD-oats, gluten free diet including oats; GFD-oats $\geqslant 8 \mathrm{~g}$, gluten free diet including at least $8 \mathrm{~g}$ of oats daily at the end of the study; GFD-std, standard gluten free diet; IEL, intraepithelial lymphocytes; EMA, antiendomysium antibodies; TGA, antitissue transglutaminase antibodies.

*Significant difference between GFD-oats and GFD-std; GFD-oats $\geqslant 8 \mathrm{~g}$ and GFD-std.

\section{Histology}

Re-examination of all initial biopsy specimens from children at the start of the study showed an enteropathy consistent with $C D$ in all cases. At the end of the one year study period, biopsy specimens were taken from all patients completing the trial. All children but two had a normal mucosal architecture (table 3). The two children with an abnormal mucosa were in the GFD-std group. When counting IEL, no significant differences were observed between the groups.

\section{Serology}

Antigliadin antibodies

Only $46 \%(47 / 102)$ of patients included in the study had IgA AGA titres above the cut off value at the time of the first diagnostic biopsy (that is, at the start of the study). All children under two years of age, except one, had AGA titres above the cut off value. Fourteen values were missing. After three months of the diet, AGA titres were below the cut off value in most children (fig 2, where only the results of children who reported consumption of at least $8 \mathrm{~g}$ of oats daily at the end of the study are shown in addition to controls $(\mathrm{n}=84) ; 55 \%(45 / 82)$ positive at the start).

\section{Antiendomysium antibodies}

At the time of the diagnostic biopsy, 94 of 100 patients (94\%) had positive IgA EMA titres. Patients who were IgA EMA negative either had IgA deficiency $(n=2)$ or were less than two years of age $(n=4)$. Sixteen values were missing. During the study year, EMA titres became negative to the same extent in both groups (fig 3). Among 92 patients that completed the study, 72\% had IgA EMA titres below the cut off value at the time of the control biopsy after approximately one year on a GFD. There was no significant difference between the two diet groups (table 2, fig 3). To determine if the titres differed between the two groups, EMA titres were

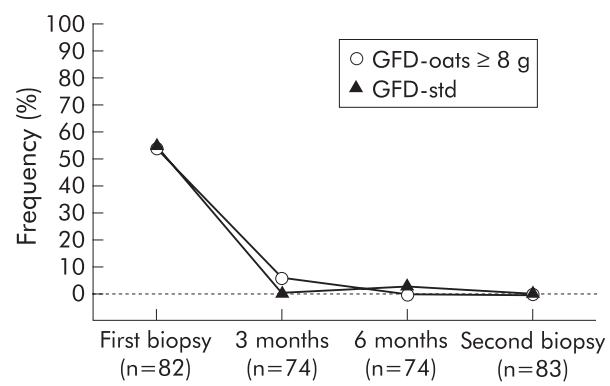

Figure 2 Frequency of positive $\lg A$ antigliadin antibodies (\%) at various checkpoints. No significant difference was found between the study groups. GFD-oats $\geqslant 8 \mathrm{~g}$, gluten free diet including at least $8 \mathrm{~g}$ of oats daily at the end of the study; GFD-std, standard gluten free diet. $n=$ the number of samples analysed. grouped as follows: 1:10-1:20 (5/34 patients in the GFD-oats $\geqslant 8 \mathrm{~g}$ group and 8/50 in GFD-std) and 1:40-1:80 (7 and 4, respectively). No significant difference was found between the groups $(\mathrm{p}=0.41)$.

\section{Antitissue transglutaminase antibodies}

The TGA assay had a lower sensitivity than the EMA assay, $90 \%(84 / 93)$ of patients having titres above the cut off value at the start of the study. Four of the IgA TGA negative patients were less than two years of age. At the end of the study, the frequency of TGA positive children among all participants was $13 \%$ (fig 4 ). There was no significant difference in TGA positivity between the groups (table 3 ). However, a significant difference was found between the quantitative median TGA titres at the end of the study, with higher TGA titres in the GFD-std group (GFD-oats $\geqslant 8 \mathrm{~g}$ median 7.0 (range 5.1-11.0); GFD-std 12.0 (5.7-15.0); $\mathrm{p}=0.04)$ but the low number of patients makes this statistic weak in power.

\section{Total serum IgA}

Two patients with total serum IgA below $0.05 \mathrm{~g} / \mathrm{l}$ were negative for all three serological markers. Both had IgG EMA titres $>1: 640$ at the start of the study. At the end of the study, they had IgG EMA titres of 1:20 and $<1: 10$, respectively.

\section{DISCUSSION}

This is the first randomised, double blind, multicentre study of the suitability of oats for children with newly diagnosed CD. Our results indicate that oats in a GFD do not prevent normalisation of the small bowel mucosa or coeliac serology markers. This is in accordance with previous open studies in a small number of children. ${ }^{17}{ }^{18}$ Similarly, absence of oats toxicity was reported in adults with $\mathrm{CD}^{7-9} 19$ and dermatitis herpetiformis. ${ }^{20}{ }^{21}$ Also, in vitro studies of duodenal biopsies

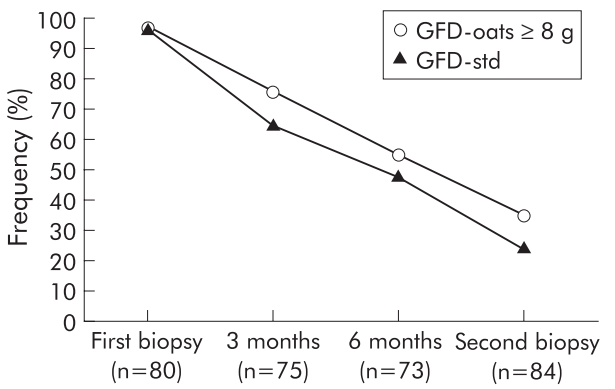

Figure 3 Frequency of positive $\lg A$ or $\lg G$ antiendomysium antibodies (\%) at various checkpoints. No significant difference was found between the study groups. GFD-oats $\geqslant 8 \mathrm{~g}$, gluten free diet including at least $8 \mathrm{~g}$ of oats daily at the end of the study; GFD-std, standard gluten free diet. $\mathrm{n}=$ the number of samples analysed. 


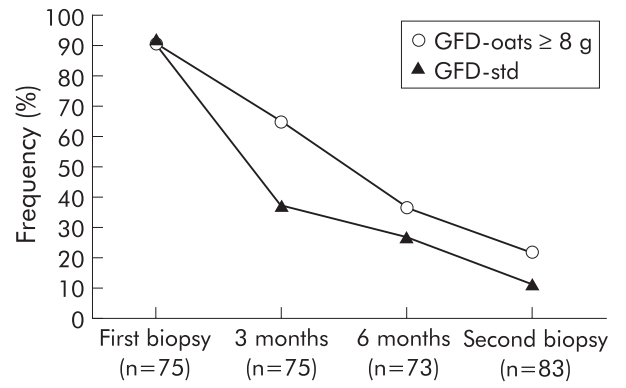

Figure 4 Frequency of positive $\lg \mathrm{A}$ antitissue transglutaminase antibodies (\%) at various checkpoints. There was a significant difference between the study groups only at the three month control. GFD-oats $\geqslant 8 \mathrm{~g}$, gluten free diet including at least $8 \mathrm{~g}$ of oats daily at the end of the study; GFD-std, standard gluten free diet. $\mathrm{n}=$ the number of samples analysed.

cultured with avenin did not reveal any immunogenicity. ${ }^{22}{ }^{23}$ It is important to note that all of these studies, including our own, presented short term results for diet test periods of up to 24 months. Absence of signs of toxicity of oats does not exclude the possibility of long term complications. ${ }^{24}$ However, Janatuinen et al performed a follow up study ${ }^{25}$ in adult coeliac patients. $^{7}$ At the time of re-examination, study patients had been on a GFD, including a daily intake of 50-70 g of oats, for five years. Compared with a control group of coeliacs on a standard GFD, there were no significant differences regarding the architecture of the duodenal mucosa or serum antibodies to endomysium, reticulin, or gliadin. The results of the study, although questioned because of the small number of patients involved, suggest that moderate amounts of oats are safe for patients with $\mathrm{CD} .^{26}$

Early reports on the tolerability of oats in patients with CD indicated a probable noxious effect on the small bowel mucosa. ${ }^{6}$ These studies were based on a small number of patients and performed without access to current antibody assays. However, Lundin and colleagues ${ }^{27}$ recently reported a case study questioning the lack of toxic effects of oats in a patient with CD. This necessitates caution and calls for further study of the long term effects of oats.

This is the first study on the effects of oats in a large population of children with newly diagnosed CD. Unfortunately, we were not able to fully achieve our aim regarding the amount of oats intake in the patients studied. This may be due to the design of the blinded study products. Some children had difficulty in consuming adequate amounts of oat products towards the end of the study year. According to a microchallenge study performed by Catassi and colleagues, ${ }^{28}$ a daily dose of $100 \mathrm{mg}$ of gliadin was enough to significantly increase the IEL count. As $100 \mathrm{mg}$ of avenin is equivalent to approximately $7.7 \mathrm{~g}$ of oats, ${ }^{2}$ we considered $8 \mathrm{~g}$ of oats daily to be the smallest quantity necessary to have a possible effect on the IEL count. However, there was no significant difference between children consuming at least $8 \mathrm{~g}$ of oats daily at the end of the study (GFDoats $\geqslant 8 \mathrm{~g}$ ) and children in the GFD-std group (table 3 ).

Withdrawal frequency was high but was not caused by the presence of coeliac symptoms in the majority of cases. When comparing the frequency of withdrawal because of symptoms between the groups of children under and over two years of age, there were significantly more children in the younger age group that withdrew $(p=0.01)$. The reason for this difference may be that small children do not tolerate oats in their diet. Whether this intolerance is due to coeliac disease and the effect of the prolamin avenin, or something else in oats, such as the high fibre content, was not investigated in this trial. Further studies are needed to investigate if there is a subgroup of coeliac patients intolerant to oats. Also, whether this inability to eat oats is immunological or due to the fibre content of the oats containing diet should be investigated.

This study was originally designed to be analysed on an intention to treat basis. However, in some of the dropout patients there was no proper follow up. Thus we lacked some vital data which makes it impossible to perform an intention to treat analysis.

It is well known that coeliac patients have great difficulties in maintaining a strict GFD. Kumar and colleagues, ${ }^{29}$ for instance, reported a dietary compliance of $44 \%$ in teenagers with $\mathrm{CD}$. One reason for this low compliance is probably the poor palatability of the comparatively low fibre standard GFD, which can be much improved by the addition of oats. It must however be emphasised that all commercial oat products used by coeliacs should be free of wheat contamination and this should be clearly marked on the packet.

In Sweden, 64\% of all small bowel biopsies performed on children with suspected CD are capsule biopsies and only $36 \%$ are performed endoscopically. ${ }^{30}$ Accordingly, the majority of small bowel biopsy specimens from coeliac children in our study were obtained by capsule. This usually permits only one or two mucosal samples to be taken, in contrast with endoscopic small bowel biopsy yielding several specimens. There is thus a theoretical possibility that biopsies in the present study could have missed patchy small bowel mucosal lesions. However, there was no significant difference in antibody titres between our study groups, which indicates lack of humoral immunological response to oats. ${ }^{22}$

In conclusion, this randomised double blind study provides evidence that the presence of moderate amounts of oats in a GFD in children with CD does not prevent clinical or small bowel mucosal healing, or humoral immunological downregulation. This finding is in accordance with previous open studies in coeliac children and adults, and indicates that oats can be safely included in a GFD for the majority of children with CD. To confirm this, long term studies of the effects of oats in coeliac children are needed.

\section{ACKNOWLEDGEMENTS}

We wish to express our sincere gratitude to the children and their parents for participating in the study, to the staff at each participating paediatric clinic, to Carina Andersson and Petra Cassel for excellent analytical work with the coeliac serology, and to dietician Mona Nilsson for invaluable help in calculating dietary intake of oats. The study was financially supported by the Cerealia Foundation R\&D, the Health Research Council in the South-East of Sweden, Swedish Nutrition Foundation, the Swedish Medical Society, Semper $\mathrm{AB}$, the Vårdal Foundation, the Odd Fellow Foundation, and Pharmacia Diagnostics, Sweden.

\section{Authors' affiliations}

L Högberg, L Stenhammar, Department of Paediatrics, Norrköping Hospital, Sweden, and Department of Paediatrics, Linköping University Hospital, Sweden

P Laurin, K Fälth-Magnusson, Department of Paediatrics, Linköping University Hospital, Sweden

C Grant, Laboratory Medicine Östergötland, Pathology, Norrköping Hospital, Sweden

E Grodzinsky, Development Unit for Primary Care and Psychiatry, County Council in Östergötland, Sweden

G Jansson, Department of Paediatrics, Motala Hospital, Sweden

H Ascher, Department of Paediatrics, The Sahlgrenska Academy, Göteborg University, Göteborg, Sweden

L Browaldh, Department of Paediatrics, Sachsska Hospital, Stockholm, Sweden

J-Å Hammersiö, Department of Paediatrics, Västervik Hospital, Sweden E Lindberg, Department of Paediatrics, Örebro University Hospital, Sweden

U Myrdal, Department of Paediatrics, Västerås Hospital, Sweden 


\section{REFERENCES}

1 Dicke WK. Coeliakie, MD Thesis Utrecht: University of Utrecht, 1950.

2 Wieser H. Prolamins in cereals. In: Lohiniemi S, Collin P, Mäki M, eds. Changing features of coeliac disease. Tampere: The Finnish Coeliac Society,
1998:25-30.

3 Vader LW, de Ru A, van der Wal Y, et al. Specificity of tissue transglutaminase explains cereal toxicity in celiac disease. J Exp Med 2002; 195:643-9.

4 Shan L, Molberg O, Parrot I, et al. Structural basis for gluten intolerance in celiac sprue. Science 2002;297:2275-9.

5 Dissanayake AS, Truelove SC, Whitehead R. Lack of harmful effect of oats on small-intestinal mucosa in coeliac disease. BMJ 1974:4:189-91.

6 Baker PG, Read AE. Oats and barley toxicity in coeliac patients. Postgrad Med J 1976;52:264-8.

7 Janatuinen EK, Pikkarainen PH, Kemppainen TA, et al. A comparison of diets with and without oats in adults with celiac disease. N Engl J Med 1995:333:1033-7.

8 Srinivasan $U$, Leonard $N$, Jones $E$, et al. Absence of oats toxicity in adult coeliac disease. BMJ 1996;313:1300-1

9 Storsrud S, Olsson M, Arvidsson Lenner R, et al. Adult coeliac patients do tolerate large amounts of oats. Eur J Clin Nutr 2003;57:163-9.

10 Collin P, Mäki M, Keyrilainen O, et al. Selective lgA deficiency and coeliac disease. Scand J Gastroenterol 1992;27:367-71.

11 Cataldo F, Marino V, Ventura A, et al. Prevalence and clinical features of selective immunoglobulin A deficiency in coeliac disease: an Italian multicentre study. Italian Society of Paediatric Gastroenterology and Hepatology (SIGEP) and "Club del Tenue" Working Groups on Coeliac Disease. Gut 1998;42:362-5.

12 Grodzinsky E, Hed J, Lieden G, et al. Presence of $\lg A$ and $\lg G$ antigliadin antibodies in healthy adults as measured by micro-ELISA. Effect of various cutoff levels on specificity and sensitivity when diagnosing coeliac disease. Int Arch Allergy Appl Immunol 1990;92:119-23.

13 Grodzinsky $E$, lvarsson $A$, Juto $P$, et al. New automated immunoassay measuring immunoglobulin A antigliadin antibodies for prediction of celiac disease in childhood. Clin Diagn Lab Immunol 2001:8:564-70.

14 Chorzelski TP, Sulej J, Tchorzewska H, et al. IgA class endomysium antibodies in dermatitis herpetiformis and coeliac disease. Ann N Y Acad Sci 1983;420:325-34.
15 Grodzinsky E, Hed J, Skogh T. IgA antiendomysium antibodies have a high positive predictive value for celiac disease in asymptomatic patients. Allergy 1994;49:593-7.

16 Marsh MN. Gluten, major histocompatibility complex, and the small intestine. A molecular and immunobiologic approach to the spectrum of gluten sensitivity ('celiac sprue'). Gastroenterology 1992;102:330-54.

17 Holm K. No harm of oats in the diet of children with newly and previously diagnosed CD. J Pediatr Gastroenterol Nutr 1998;26:549.

18 Hoffenberg EJ, Haas J, Drescher A, et al. A trial of oats in children with newly diagnosed celiac disease. J Pediatr 2000;137:361-6.

19 Janatuinen EK, Kemppainen TA, Pikkarainen PH, et al. Lack of cellular and humoral immunological responses to oats in adults with coeliac disease. Gut 2000;46:327-31.

20 Hardman CM, Garioch JJ, Leonard JN, et al. Absence of toxicity of oats in patients with dermatitis herpetiformis. N Engl J Med 1997;337:1884-7.

21 Reunala T, Collin P, Holm K, et al. Tolerance to oats in dermatitis herpetiformis. Gut 1998:43:490-3.

22 Picarelli A, Di Tola M, Sabbatella L, et al. Immunologic evidence of no harmful effect of oats in celiac disease. Am J Clin Nutr 2001;74:137-40.

23 Kilmartin C, Lynch S, Abuzakouk M, et al. Avenin fails to induce a Th1 response in coeliac tissue following in vitro culture. Gut 2003;52:47-52.

24 Branski D, Shine M. Oats in celiac disease. N Engl J Med 1996;334:865-6.

25 Janatuinen EK, Kemppainen TA, Julkunen RJ, et al. No harm from five year ingestion of oats in coeliac disease. Gut 2002;50:332-5.

26 Dor R, Shanahan DJ. Oats and coeliac disease. Gut 2002;51:757-8.

27 Lundin KEA, Nilsen EM, Scott HG, et al. Oats induced villous atrophy in coeliac disease. Gut 2003;52:1649-52.

28 Catassi C, Rossini M, Ratsch IM, et al. Dose dependent effects of protracted ingestion of small amounts of gliadin in coeliac disease children: a clinical and jejunal morphometric study. Gut 1993;34:1515-9.

29 Kumar PJ, Walker-Smith J, Milla P, et al. The teenage coeliac: follow up study of 102 patients. Arch Dis Child 1988;63:916-20.

30 Stenhammar L, Ascher H, Danielsson L, et al. Small bowel biopsy in Swedish paediatric clinics. Acta Paediatr 2002;91:1126-9. 